\title{
Non-medical aspects of civilian-military collaboration in management of major incidents
}

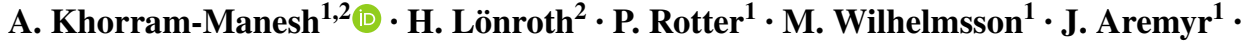 \\ A. Berner ${ }^{1} \cdot$ A. Nero Andersson ${ }^{1} \cdot$ E. Carlström ${ }^{1,3}$
}

Received: 23 November 2016 / Accepted: 21 February 2017 / Published online: 2 March 2017

(C) The Author(s) 2017. This article is published with open access at Springerlink.com

\begin{abstract}
Purpose Disasters and major incidents demand a multidisciplinary management. Recent experiences from terrorist attacks worldwide have resulted in a search for better assessment of the needs, resources, and knowledge in the medical and non-medical management of these incidents and also actualized the need for collaboration between civilian and military healthcare. The aim of this study was to evaluate the impact of the civilian-military collaboration in a Swedish context with the main focus on its non-medical management.

Method An exercise, simulating a foreign military attack centrally on Swedish soil, was designed, initiated, and conducted by a team consisting of civilian and military staff. Data were collected prospectively and evaluated by an expert team.

Results Specific practical and technical issues were presented in collaboration between civilian and military staffs. In addition, shortcomings in decision-making, follow-up, communication, and collaboration due to prominent lack of training and exercising the tasks and positions in all managerial levels of the hospital were identified.

Conclusion Current social and political unrests and terror attacks worldwide necessitate civilian-military
\end{abstract}

A. Khorram-Manesh

Amir.khorram-manesh@surgery.gu.se

1 Prehospital and Disaster Medicine Center, Regionens Hus, 40544 Gothenburg, Sweden

2 Department of Surgery, Institute of Clinical Sciences, Sahlgrenska Academy, Gothenburg University, Gothenburg, Sweden

3 Department of Health and Crisis Management and Policy, Sahlgrenska Academy, Gothenburg University, Gothenburg, Sweden collaboration. Such collaboration, however, needs to be synchronized and adjusted to avoid preventable medical and non-medical consequences. Simulation exercises might be one important source to improve such collaboration.

Keywords Civilian-military $\cdot$ Trauma $\cdot$ Terrorism · Collaboration $\cdot$ Simulation $\cdot$ Exercise

\section{Introduction}

The increasing risk of natural disasters, as well as, major incidents created by human, such as terror attacks, indicates a need for collaboration between different agencies [1, 2]. In 2010, during the devastating flooding, which killed 2000 people and affected 20 million people, the Pakistani military troops were mobilized to collaborate and assist the civilian agencies, e.g., healthcare. They accomplished immediate life-saving assistance, including rations from the military's supplies as well as field hospitals and relief camps. Other examples of civilian-military (CM) collaboration are Thailand during the Indian Ocean tsunami 2004, the 2005 Pakistan earthquake, and the 2010 Haiti earthquake [1].

Recent experiences from terrorist attacks worldwide, particularly in Europe (France 2015), have resulted in a search for better assessment of the needs, resources, and knowledge in the medical and non-medical management of these incidents $[2,6]$. As the attacks vary in type, magnitude, and outcomes, and result in injuries, which are rarely treated in a civilian setting, the needs for a wider planning and CM healthcare collaboration is obvious. The CM collaboration in the medical management of victims is often high lightened and mentioned as one of the major cause of a successful outcome [2-7]. 
Although CM collaboration is common in battle areas, especially within the field of healthcare, relatively few studies have scrutinized the effects of such collaboration from a civilian perspective $[4,5]$. In all examples of overwhelming mass casualties, the civilian society is in dire need of support from military resources. However, the opposite situation may exist, i.e., the military might be in need of civilian healthcare resources during a military action. An assumption in such situation would be that such collaboration puts the civilian medical community and trauma centers into a test. The challenges would be both medical and non-medical. The medical challenges would be: the knowledge of damage control management at prehospital and hospital levels; the ability to treat immediate survivors with military injury patterns; the outcome of such treatment in terms of mortality and morbidity; etc [2, 5-10]. The nonmedical issues, such as command and control, communication, collaboration, logistic, information etc, are fairly well discussed in the literature, but few studies have prospectively analyzed the impact of a CM collaboration in respect to these topics $[3,4,6]$. Some of the issues might be solved based on the facts and knowledge, and some by relying on the experience and background of involved managers at operational and tactical levels [11].

To analyze the non-medical outcomes of a CM collaboration, the focus should mainly be on the organizational and logistic interface of the $\mathrm{CM}$ healthcare. Although there are many guidelines, which regulate $\mathrm{CM}$ collaboration, they are not adopted to conflicts in western countries. The Oslo guidelines developed in 1994 and updated in 2006 address only natural disasters in times of peace. The MDCA guidelines (guidelines on the use of military and civilian assets to support UN humanitarian activities in complex emergencies) from 2003 are developed to suit humanitarian actions in countries, such as Iraq and Afghanistan, where the foreign military has been in the presence [12].

The aim of this paper was to analyze the outcomes of CM collaboration in a mutual exercise, in which military casualties were brought to a civilian hospital for further care. The focus was mainly on the surge capacity and organizational structure, command and control, participants knowledge about their responsibilities, functions, and organizational belonging during a major incident, their ability of understanding the nature of issues that may appear during an incident, such as triage of resources, organizational maintenance, safety and communication, logistic, and the need and demands for developing CM collaboration.

The scenario used in this exercise was a foreign military attack centrally on the western region of Sweden. A prominent industrial zone in Sweden with 1.6 million inhabitants, living in urban as well as rural, and scarcely populated areas with five major hospitals with emergency departments (ED) and emergency care competency [13]. Complementary care in some specialties (e.g., Neurosurgery, vascular surgery, etc) is provided by the university hospital in Gothenburg; the only trauma center in the region. The attack generated a varying number of casualties $(n=28)$ with typical war injuries. They were transported by military helicopters, either as single victims or two on the same helicopter, from the field hospital/s to the trauma center in Gothenburg. They were all landed on the helipad and delivered to the civilian staff after a report exchange and were admitted through the ED on Monday 31 August 2015, starting at 08:00 a.m.

\section{Method and material}

\section{Evaluation template and observations}

Data were collected prospectively using an evaluation template based on CSCATTT [14]. This template consisted of the following sections: Command and control, Safety, Communication, Assessment, Triage, Treatment, and Transport were reviewed and evaluated by free text. Observers were allowed to comment all activities, pros, and cons and if possible, and evaluate any specific activity by giving a point between 1 and 10 on a VAS scale $(10=$ excellent $)$.

During the observations, all observers accompanied and observed the military and hospital employees at different stations (see in the following) [15]. The raw data could be collected, even though the event itself proceeded relatively fast [16]. The analysis was carried out in three steps. First, we selected the relevant effects of military-civil integration within the exercise. In the second step, we coded the findings. Then, we sorted and analyzed all the observations as a whole [17].

A total number of nine observers/evaluators were engaged in the evaluation of scenario and were localized at the following station;

Helipad The place for first communication between military and civilian staff.

Transportation path From helipad to ED

$E D$ The first place to examine, triage, and manage all victims at admission and trauma bay

Hospital command group (HCG) A group of decision makers, with local responsibilities to organize, assess, and maintain the local needs at the hospital.

The total time of observations was $63 \mathrm{~h}$.

\section{Results}

The results indicating the shortcomings and the points for improving are given for each station. Since some of the 
issues may be related to the shortcomings in hospitals contingency plan and not $\mathrm{CM}$ collaboration, all issues were divided into issues related to $\mathrm{CM}$ collaboration (A) and those related to hospitals contingency plan (B).

\section{Helipad}

\section{Issues related to CM collaboration}

Reporting system Different reporting systems between military and civilian healthcare staff were identified. The military staff used ATMIST (Age of the patient, Time of the incident, Mechanism of injury, Injury, Signs, Treatment) or MIST (Mechanism of injury, Injury, Signs, Treatment), while civilian staff used SBAR (Situation, Background, Actual, Recommendations). This discrepancy in reporting resulted in misunderstanding, low situation awareness, missing vital patient information, and initiation of unnecessary measures. Five important points in the process of reporting (identifying right person to report to, silent minute to absorb the information and let the report flow, content of the report, standardization of the report and documentation) were particularly noted by observer/evaluator in ten randomly chosen red patients and were given a point on the
VAS scale, as mentioned in "Method" (Table 1). The lack of standardization in the reports and a proper documentation were prominent.

Protocol There was no standardized protocol for reporting the patient's medical history and/or medical signs between military and civilian staffs. This resulted in a hand-written report, which was time-consuming, hard to read and lacked vital information (Fig. 1).

Noise In comparison with a civilian helicopter, a military helicopter never turns off its motor as practiced during the war. Consequently, the whole report was given under functioning helicopter and created a noisier environment in which key information was lost.

Military stretchers Patients were carried on military stretchers, which were supposed to be changed at the time of patient delivery with a similar one at the hospital. However, the military and civilian stretchers were not compatible, and therefore, the military stretchers had to be returned. The process of off-loading patients from military stretchers and re-loading to a civilian stretcher was time-consuming and jeopardized the patient safety.
Table 1 Military-to-civilian reporting process on the helipad in ten randomly chosen red patients

\begin{tabular}{lccrrrrrrrrr}
\hline Patient & $\mathrm{A}$ & $\mathrm{B}$ & $\mathrm{C}$ & $\mathrm{D}$ & $\mathrm{E}$ & $\mathrm{F}$ & $\mathrm{G}$ & $\mathrm{H}$ & $\mathrm{I}$ & $\mathrm{J}$ & Average \\
\hline $\begin{array}{l}\text { Identifying the right } \\
\quad \text { person to report }\end{array}$ & 2 & 6 & 8 & 8 & 8 & 8 & 10 & 8 & 10 & 10 & 7.6 \\
$\quad$ Silence minute & 2 & 6 & 10 & 10 & 10 & 10 & 10 & 10 & 10 & 10 & 8.8 \\
Content of the report & 4 & 6 & 8 & 8 & 8 & 8 & 10 & 8 & 8 & 10 & 7.8 \\
Standardized report & 2 & 4 & 4 & 4 & 4 & 4 & 8 & 4 & 4 & 8 & 4.2 \\
Documentation & 4 & 4 & 6 & 6 & 6 & 6 & 6 & 6 & 4 & 6 & 5.4 \\
\hline
\end{tabular}

$10=$ excellent
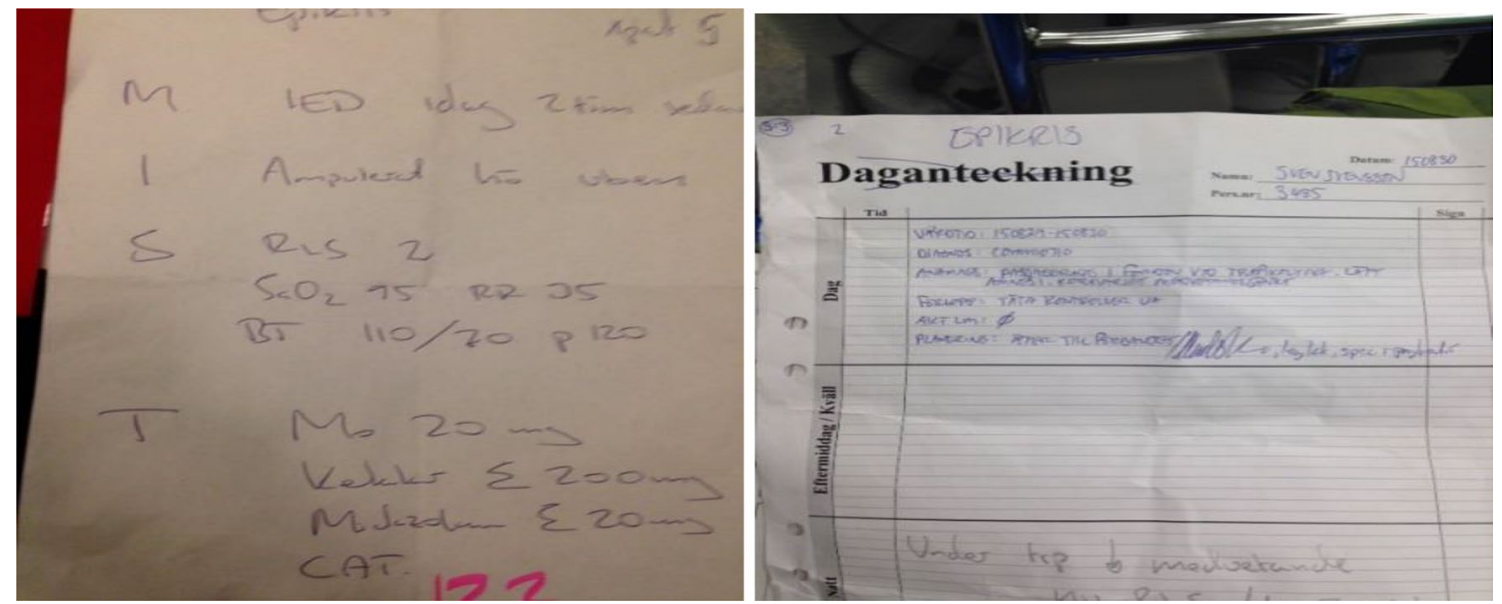

Fig. 1 Readability of the hand-written report 


\section{Issues related to contingency plan}

Wind During the exercise, it was very difficult to hold loose items, including triage tags, medical items, papers, etc, due to the winds and the turbulence from the helicopter. There was no covering area, which could protect patients and staff from rain, wind, and cold.

\section{Hospital logistic; transport path to the ED}

\section{Related to CM collaboration}

Medical resources Due to the high number of casualties and consequent multiple helicopter landings, staff and instrument were not in place all the time. With each nurse leaving the scene, her replacement could delay, since they used the same path. In a daily and customary basis, there would be few helicopter arrivals, which can easily be handled.

Reporting times The time for giving report was extremely short at the trauma bay (15 s) compared to report given on helipad (45 s). There were obvious problems with the presentation of the hand-written report from the helipad area to the trauma team (Table 2). Looking at the same parameters in the process of reporting (identifying right person to report to, silent minute to absorb the information and let the report flow, content of the report, and standardization of the report and documentation); beside the lack of standardization and documentation, the scarce content of the report was also prominent.

\section{Related to contingency plan}

\section{Transport time to the ED}

Table 3 shows the time from helicopter landing to the time when the patient was reported to the trauma room. The transport time varied between 9 and 16 (average 11.9) $\mathrm{min}$. Delays occurred if elevators were not running fast. The time was much longer if two patients were delivered by the military helicopter. The table shows the time consumed for ten randomly chosen patients at different levels from landing to the end of reporting at trauma bay. The last column shows the average of consumed time in each level.

\section{Competency during patient transportation}

Almost all patients were transported by a single nurse together with another caretaker with no medical knowledge. Any deviation in patients' vital signs could not be medically responded easily with only one nurse available.

\section{Communication}

The phone used by the nurse stopped working in periods during transportation and mainly in the elevator, thus leaving the sole nurse alone should anything severe happen. In eight out of ten victims, triaged as severe cases
Table 2 Reporting time consumed in civilian-civilian reporting process for the patients in this study
Table 3 Time from helicopter landing until the patient is delivered to the ED

\begin{tabular}{lrrrrrrrrrrr}
\hline Patient & $\mathrm{A}$ & $\mathrm{B}$ & $\mathrm{C}$ & $\mathrm{D}$ & $\mathrm{E}$ & $\mathrm{F}$ & $\mathrm{G}$ & $\mathrm{H}$ & $\mathrm{I}$ & $\mathrm{J}$ & Average \\
\hline $\begin{array}{l}\text { Identifying the person who } \\
\quad\end{array}$ & 10 & 10 & 10 & 10 & 10 & 10 & 10 & 10 & 10 & 10 & 10 \\
$\quad$ receives the report & & & & & & & & & & & \\
Silence minute to let report flow & 10 & 10 & 10 & 10 & 10 & 10 & 10 & 10 & 10 & 10 & 10 \\
Content of the report & 4 & 4 & 4 & 8 & 6 & 8 & 8 & 1 & 6 & 6 & 5.5 \\
Standardized report & 0 & 0 & 0 & 0 & 0 & 0 & 0 & 0 & 4 & 0 & 0.4 \\
Documentation & 6 & 2 & 6 & 6 & 4 & 6 & 4 & 2 & 4 & 4 & 4.4 \\
\hline
\end{tabular}

$10=$ excellent

\begin{tabular}{llllllllllll}
\hline Patient & A & B & C & D & E & F & G & H & I & J & Average \\
\hline Helicopter lands & 0 & 0 & 0 & 0 & 0 & 0 & 0 & 0 & 0 & 0 & 0 \\
Start reporting on helipad & +4 & +4 & +5 & +2 & +3 & +4 & +5 & +3 & +3 & +4 & 3.7 \\
End reporting on helipad & +1 & +0 & +4 & +1 & +0 & +1 & +1 & +1 & +1 & +0 & 1.0 \\
At the elevator (helipad) & +3 & +2 & +2 & +1 & +1 & +3 & +1 & +1 & +1 & +1 & 1.6 \\
Arrival at ED & +4 & +4 & +4 & +4 & +4 & +5 & +5 & +4 & +4 & +5 & 4.3 \\
Start reporting at trauma bay & +1 & +1 & +1 & +1 & +1 & +1 & +1 & +2 & +1 & +1 & 1.1 \\
End reporting at trauma bay & +0 & +0 & +0 & +1 & +0 & +0 & +0 & +0 & +1 & +0 & 0.2 \\
Total time (min) & 13 & 11 & 16 & 10 & 9 & 14 & 13 & 11 & 11 & 11 & 11.9 \\
\hline
\end{tabular}


(red tags), there was a need for additional qualified staff during transportation to maintain the high quality and ensure the safety of patients during transportation.

\section{ED (emergency department)}

\section{Related to CM collaboration}

None.

\section{Related to contingency plan}

Teamwork and organization Good situation awareness, initially common understanding of the incident, review of the mass casualty plan, and good preparation. However, with the arrival of victims, ED was transferred to a more disorganized area, stepwise, e.g., some of the staff could not find the way to the helipad. Traffic jam at the entrance and exit of the trauma bay area. There were two doors, but just one was used.

Information There was a misunderstanding about the number of casualties in many occasions, especially after a while when the number of receiving patients did not match the number of existing patients. The information given to the staff at trauma bay was scarce and many wondered where the patient come from? No one realized that the victims came from field hospitals. There was a lack of information about, where the victims went to and with which medical files? The exercise was over, however, no one knew who made the decision, when and why?

Communication Wrong numbers on the list delay the arrival of the surgeon and anesthesiologist. No connection to the emergency laboratory probably due to the network problem. Communication problem between staff on the helipad, ED, and intensive care unit concerning where to patients should be transported.

Documentation Only one secretary worked with documentation at trauma bay and she was overloaded. There was also a confusion about which admission template should be used, the ordinary one or the one designated for disaster?

Resources Neck stabilizing collars in the trauma bay were not enough.

The performance of ED at two levels, outside and inside trauma bay, based on a preplanned template with specific topics, is given in detail in Table 4. Points were given based on the VAS scale (Table 4).

\section{Hospital command group (HCG)}

\author{
Related to CM collaboration
}

None.

\section{Related to contingency plan}

Teamwork and organization Smooth teamwork early in the process, in designated room, based on the hospitals' disaster and contingency plan, in a seemingly well-equipped room.

Communication Problem, especially concerning the level of preparedness.

Information They had a timely press release. However, it was unclear if the amount and content of released information were known to the public, emergency medical services, others emergency departments, primary care, as well as internally?

Follow-up Decision made or scenarios discussed were not followed up.

Instruments Problem with conference and communication devices sporadically.

The performance of HCG, based on a preplanned template with specific topics, is given in detail in Table 5 . Points were given based on the VAS scale (Table 5).

\section{Discussion}

A successful CM collaboration in the medical management of major incidents relies not only on the skills and knowledge of the staff but also on non-medical compatibility and harmonization of both organizations. Maybe, the strongest message sent by this study is that any kind of multidisciplinary management should be harmonized and tested for compatibility by mutual planning, training and exercising $[2,18-21]$.

Different countries have different health care systems that may impact the military involvement in civilian disasters and civilian involvement in military conflicts [2]. Some countries have military hospitals, which treat military staff and their families in peacetime and military casualties during the war, and thus, military victims will not be treated in the civilian sector, e.g., USA. Other countries have no military hospitals and civilian hospitals are responsible for the treatment of military casualties, e.g., Israel. In Sweden, there are no military hospitals and emergency care is offered to the staff through full-time or part-time employed 
Table 4 Evaluation of ED's organizational structure in VAS scale

\begin{tabular}{|c|c|c|}
\hline & Points & Comments \\
\hline \multicolumn{3}{|l|}{ Outside trauma bay } \\
\hline Obtaining information & 5 & \\
\hline Analyzing ability & 5 & \\
\hline Decision-making ability & 8 & No follow-up and analysis \\
\hline Follow-up a decision & 6 & See above \\
\hline Team/organizational build-up & 9 & Initially good, but worsen with chaos \\
\hline Establishing contact with commanders & 9 & Excellent \\
\hline Working areas & 5 & Not good for commanding \\
\hline Resources & 6 & Scarce \\
\hline Individual equipment & 8 & Some lacking \\
\hline Information sharing & 5 & Unclear sometimes \\
\hline Cooperation between physicians & 9 & \\
\hline Identifying receiver of the report & 6 & Who needs the information and how? \\
\hline Silence minute & 1 & None \\
\hline Standardized reporting template & 1 & None \\
\hline Written information & 5 & Not complete e.g. alerting level \\
\hline Assessment of the situation & 5 & Number of patients vs available beds \\
\hline Communication with other units & 7 & \\
\hline \multicolumn{3}{|l|}{ Inside trauma bay } \\
\hline Team/organization build-up & 7 & \\
\hline Admission of patients & 4 & \\
\hline Working area & 7 & Long distance to helipad and intensive care unit \\
\hline Available resources & 8 & Not realistic, hard to measure \\
\hline Management of patients & 8 & ATLS-based \\
\hline Identifying receiver of information & 8 & \\
\hline Silence minute & 7 & Most of the time \\
\hline Contents of the report & 5 & Large variation \\
\hline Report template & 3 & None \\
\hline Situation assessment & 9 & \\
\hline ATLS guidelines & 9 & Yes \\
\hline Patients transported to known space & 9 & Yes \\
\hline Teamwork & 9 & Yes \\
\hline Equipment & 9 & Yes \\
\hline
\end{tabular}

Ten points $=$ excellent military physicians and nurses in collaboration with the university hospitals. If necessary, patients can be referred to the hospitals for further assessment, treatment, and surgical interventions. During an armed conflict or war, Swedish army relies fully on the civilian resources [22].

The collaboration between civilian and military healthcare has long been discussed and recommended mainly due to the civilian healthcare's economic austerity and its need for sharing resources in terms of manpower and medical devices, vehicles, etc. A new and important reason is the increasing number of terror attacks in last decade with new patterns of injuries (gunshot and explosives) that are totally new to the civilian healthcare $[2,6]$. With an increasing number of social disturbances encumbering many countries and putting them in an everyday alertness, any involvement in an armed conflict implicates another burden to their healthcare that must be prepared for [2, 13, 23-27]. Such preparedness should be multidisciplinary planned, tested and conducted [18-20].

It is already known that the medical management of mass casualties needs special consideration and calls for a CM collaboration [2]; however, the non-medical collaboration should also be prepared. In this study, by dividing our findings into $\mathrm{CM}$ collaboration-related and contingency plan-related issues, we could identify those issues that particularly should be addressed within the CM collaboration framework.

There was no surprise that most of the issues in the interface of CM collaboration occurred in the area, where they meet for the first encounter, i.e., helipad and during 
Table 5 Evaluation of HCG in VAS scale points 1-10

\begin{tabular}{|c|c|c|}
\hline Action & Point & Comments \\
\hline Obtaining information & 7 & $\begin{array}{l}\text { Was received through telephone calls and meetings. However, information obtained was } \\
\text { not shared adequately }\end{array}$ \\
\hline Analyzing ability & 7 & High, but not in all functions \\
\hline Decision-making ability & 8 & Very high, but not in all functions \\
\hline Follow-up of decisions made & 5 & $\begin{array}{l}\text { No clear follow-up routine including unclear distribution of tasks. Lack of visual over- } \\
\text { view of what, who, when and how? }\end{array}$ \\
\hline Teamwork/organizational form & 7 & $\begin{array}{l}\text { Good, including participation of all members in discussion, assessments, and suggestions } \\
\text { Supporting each other in an overloaded situation } \\
\text { Good knowledge about self- and each other's role and good interaction }\end{array}$ \\
\hline Cooperation & 7 & Good \\
\hline Coordination & 7 & Good \\
\hline Communication & 6 & $\begin{array}{l}\text { Confusing } \\
\text { Technically problematic }\end{array}$ \\
\hline Identifying report receiver & 7 & Very good \\
\hline Reporting template & 1 & None \\
\hline Written information & 4 & Not in all occasions \\
\hline Situation assessment & 9 & Excellent \\
\hline
\end{tabular}

$10=$ excellent

the transportation. Issues such as functioning helicopter during the reporting, non-compatible stretchers, different reporting systems, not only jeopardize patient's safety but also creates unnecessary and time-consuming measures, which have an impact on the outcome of whole management. The experience gained from recent attacks in France is good enough to realize that a harmonized multidisciplinary approach is a necessity in the management of disasters and major incidents [2,6]. Standardization is possible by introducing mutual protocols, training, and guidelines [20].

Other issues found in this report were directly related to the hospital and its contingency plan. The long transport path to the ED (256 m of underground culverts, including two elevators) is all structural issues that may jeopardize patient safety. Although the hospital had a valid and comprehensive plan, the people using it were not trained and familiar with the operational items. The lack of training and exercising the tasks and positions was prominent in this study and in different positions (e.g., how to perform the tasks, how to find the way to different sections, how to use various routines, how to identify the right partner for collaboration, and how to analyze and react to unplanned issues). This cannot be done by just a written text in a plan, and a good preparedness is achieved by being exposed to the possible event, new scenarios, exercises, and training $[20,21,24]$.

To maintain a high level of preparedness, special considerations should be paid to the preparedness pyramid, which identifies planning, infrastructure, knowledge, and capabilities as the major components [26]. Since all Swedish hospitals have both disaster plans and good infrastructure, the exercise itself gave us a chance to evaluate the level of knowledge and capabilities and thus complete the preparedness pyramid. Studying command and control, communication and collaboration at the hospital, the HCG showed good ability in commanding, group dynamic, and teamwork. However, they were not strong enough in control, communication, and collaboration with other agencies or units. One of the major problems was the inability of following up various decisions or plans, which were made and discussed. Alongside the good discussion and democratic decision-making, it was often forgotten to point out a specific individual to follow a specific task and order [11, 23, 27-29].

Communication internally and externally was also weak and may be a reason why collaboration with other agencies was not in focus. Communication network was overloaded quickly. The process of paging different physicians did not work during the exercise. Overloaded telephone network and other problems with IP telephony have been emphasized in many studies. The former was recently reported in the terrorist attack in Brussels [2, 13, 25].

The main goal for evaluation of ED was to assess the organizational structure and not the medical assessment of the victims. All patients were medically assessed by an experienced surgical team at trauma bay and based on ATLS guidelines. It is, however, important to emphasize that exercises with no real-time treatment schedule, with or without figurants or patient cards, cannot generate measurable parameters and thus are not fully evaluable. 


\section{Limitations}

In this study, an observational method was used to study the exercise [15-17]. The main advantage of observing exercises is twofolds: (1) the possibility of following the event directly as it happens and (2) the documentation of the participant's behavior from the arrival of the first unit until the situation is normalized and the exercise has ended [18-21]. This method is more favorable than a retrospective study in which post-event data collection can be difficult and the events can be viewed as vague reconstructions. In addition, the participants often have difficulty remembering exactly what happened, in what order things were done, and who did what $[23,28,29]$. The limitation with this method is, however, the observer himself, since the evaluation of the exercise is based on evaluator's background, experience, and his/her ability to picture the whole working process without any involvement and in a passive stance. Using free text as comment often express an individual perception and may also be individually comprehended.

Although we used VAS scale to measure some parameters, the points given are also individual-based. Some studies recommend measurable parameters and performance indicators such as time consumed with different actions [27]. However, a quick decision does not necessarily equal the right and best solution.

A better evaluation would be possible if the whole chain of actions, including prehospital care, triage, transport to the ED and from ED to the ward, could be involved. This would probably show even weaknesses at the prehospital level with impacts on the decision made at the hospital.

\section{Conclusion}

In conclusion, the immediate response to a major incident such as a terror attack is a combination of medical and nonmedical measures in a multidisciplinary manner. Although quick and appropriate medical intervention is vital for the survival of victims, the non-medical measures, such as establishing command and control, safety, proper communication, collaboration with other agencies, information, logistic, etc, are equally important. Any kind of multidisciplinary approach, including CM collaboration, should be harmonized by mutual planning, training, and exercising.

Acknowledgements AK designed the evaluation and wrote the manuscript. HL initiated and designed the exercise. EC supervised the exercise. All authors read, improved, and approved the manuscript. Authors would also like to thank all involved civilian and military staff for their participation.

\section{Compliance with ethical standards}

Conflict of interest Amir Khorram-Manesh, Hans Lönroth, Pontus Rotter, Mikael Wilhelmsson, Johan Aremyr, Andreas Berner, Agneta Andersson Nero, and Eric Carlström declare no conflict of interest.

Open Access This article is distributed under the terms of the Creative Commons Attribution 4.0 International License (http:// creativecommons.org/licenses/by/4.0/), which permits unrestricted use, distribution, and reproduction in any medium, provided you give appropriate credit to the original author(s) and the source, provide a link to the Creative Commons license, and indicate if changes were made.

\section{References}

1. Madiwale A, Virk K. Civil-military relations in natural disasters: a case study of the 2010 Pakistan floods. IRRC. 2011;93(884):1085-105.

2. Khorram-Manesh A. Europe on fire; medical management of terror attacks-new era and new considerations. Bull Emerg Trauma. 2016;4(4):183-85.

3. Etkin D, McBey K, Trollope C. The military and disaster management: a Canadian perspective on the issue, Canadian risk and hazards network. http://www.crhnet.ca/sites/default/files/library/ Etkin.pdf. Accessed 2 Mar 2017.

4. Marklund L, Graham AM, Morton PG, Hurst CG, et al. Collaboration between civilian and military healthcare professionals: a better way for planning, preparing, and responding to all hazard domestic events. Prehosp Disaster Med. 2010;25(5):399-412.

5. Frykberg ER. Medical management of disasters and mass casualties from terrorist bombings: How can we cope? Trauma. 2002;53(2):201-12.

6. Hirsch M, Carli P, Nizard R, Riou B, et al. The medical response to multisite terrorist attacks in Paris. Lancet. 2015;386:2535-38.

7. Frykberg ER. Terrorist bombings in Madrid. Crit care. 2004;9:20.

8. de Ceballos JPG, Turégano-Fuentes F, Perez-Diaz D, SanzSanchez M, et al. 11 March 2004: The terrorist bomb explosions in Madrid, Spain - an analysis of the logistics, injuries sustained and clinical management of casualties treated at the closest hospital. Crit Care. 2004;9:104.

9. Gates JD, Arabian S, Biddinger P, Blansfield J, et al. The initial response to the Boston marathon bombing: lessons learned to prepare for the next disaster. Ann Surg. 2014;260:960-66.

10. Tourtier JP, Palmier B, Tazarourte K, Raux M, et al. The concept of damage control: extending the paradigm in the prehospital setting. Ann Fr Anesth Reanim. 2013;32:520-26.

11. Khorram-Manesh A, Berlin J, Carlström E. Two validated ways of improving the ability of decision-making in emergencies. Results from a literature review. Bull Emerg Trauma. 2016;4(4):186-96.

12. OCHA. Oslo Guidelines. Guidelines on the use of foreign military and civil defence assets in disaster relief. https://docs.unocha.org/sites/dms/Documents/Oslo\%20Guidelines\%20ENGLISH\%20(November\%202007).pdf. Accessed 2 Mar 2017.

13. Khorram-Manesh A, Hedelin A, Örtenwall P. Regional coordination in medical emergencies and major incidents; plan, execute, and teach. Scand J Trauma Resusc Emerg Med. 2009;17:32.

14. MIMMS (Major Incident Medical Management and Support. http://www.alsg.org/uk/MIMMS. Accessed 2 Mar 2017.

15. Spradley JR. The ethnographic interview. http:// alexlinsker.com/101/wp-content/uploads/2010/08/ 
The-Ethnographic-Interview-by-JP-Spradley-c1979.pdf. Accessed 2 Mar 2017.

16. Burgess C, Cornelius V, Love S, Graham J, et al. Depression and anxiety in women with early breast cancer; a five year observational cohort study. BMJ. 2005;330:702.

17. Pope C, Mays N. (eds) Qualitative research in health care. 2006. BMJ Books, London.

18. Lateef F. Simulation-based learning: just like the real thing. J Emerg Trauma Shock. 2010;3(4):348-52.

19. Kim H. Improving simulation exercises in Korea for disaster preparedness. Disaster Prev Manag. 2013;22(1):38-47.

20. Khorram-Manesh A, Lupesco O, Friedl T, Arnim G, et al. Education in disaster management: what do we offer and what do we need? Proposing a New Global Program. Disaster Med Public Health Prep. 2016;20:1-20 (epub ahead of print).

21. Khorram-Manesh A. Training in disaster medicine and emergencies; a short review. Austin J Emerg Crit Care Med. 2015;2(4):1024.

22. Swedish Armed Forces. Military healthcare. http://www.forsvarsmakten.se/en/. Accessed 2 Mar 2017.
23. Sikström S. Forgetting curves: implications for connectionist models. Cogn Psychol. 2002;45(1):95-152.

24. Carter C. Managing a major incident in the critical care unit. Nurs Stand. 2013;28(31):39-44.

25. Khorram-Manesh A, Hedelin A, Örtenwall P. Hospital-related incidents; causes and its impact on disaster preparedness and prehospital organisations. Scand J Trauma Resusc Emerg Med. 2009; 17: 26.

26. Adini B, Goldberg A, Laor D, Cohen R, et al. Assessing levels of hospital emergency preparedness. Prehosp Disaster Med. 2006;21(6):451-57.

27. Nilsson H, Rüter A. Management of resources at major incidents and disasters in relation to patient outcome: a pilot study of an educational model. Eur J Emerg Med. 2008;15(3):162-65.

28. Vadillo MA, Kostopoulou O, Shanks DR. A critical review and meta-analysis of the unconscious thought effect in medical decision making. Front Psychol. 2015;6:636.

29. Manigault AW, Handley IM, Whillock SR. Assessment of unconscious decision aids applied to complex patient-centered medical decisions. J Med Internet Res. 2015;17(2):e37. 